Revista del Centro de Investigación de la Universidad La Salle

Vol. 14, No. 55, Enero-Junio, 2021: 81-102

DOI: http://doi.org/10.26457/recein.v14i55.2773

\title{
Impacto de la pandemia Covid-19 sobre profesores y estudiantes en escuelas de negocios en México
}

\author{
Impact of Covid-19 pandemic on professors and students in \\ business schools in Mexico
}

\author{
Carlos Gabriel Colín Flores \\ Universidad Anáhuac México (México)
}

Recibido: 12 de septiembre de 2020

Aceptado: 9 de marzo de 2021

Publicado: 31 de mayo de 2021

\section{Resumen}

El objetivo de este estudio es el entendimiento del impacto que tiene la pandemia de Covid-19 en la salud mental de estudiantes y profesores de escuelas de negocios en México, mediante la evaluación de las variables: depresión, ansiedad, insomnio, impacto del evento Covid-19 y agotamiento.

Para este propósito, se realizó un estudio longitudinal con 834 participantes (161 profesores y 673 estudiantes), se calcularon las correlaciones entre las variables de estudio, un análisis de frecuencias y posteriormente un análisis de varianza teniendo como variable independiente a la presencia o no de la pandemia -inicio y final del curso-, como variable moderadora, el tipo de participante -estudiante o profesor- y la variable dependiente, la variable relacionada con la salud mental en cuestión.

Las conclusiones indican que hay un impacto en la salud mental de los participantes derivado de la pandemia de Covid-19 y que éste es diferente estudiantes y profesores, siendo estos últimos los más afectados en su salud mental por los efectos de la pandemia Covid-19.

Palabras clave: Salud mental, depresión, ansiedad, insomnio, agotamiento

*Email: carlos colin01@prodigy.net.mx

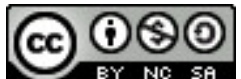

Revista del Centro de Investigación. Universidad La Salle por Dirección de Investigación. Universidad La Salle Ciudad de México se distribuye bajo una Licencia Creative Commons Atribución-NoComercial-CompartirIgual 
Colín Flores, C. G.

\section{Abstract}

The purpose of this study is to understand the impact of Covid-19 pandemic on mental health of students and professors in business schools in Mexico, through the evaluation of variables: depression, anxiety, insomnia, impact of event Covid-19 and burnout.

A longitudinal study was done, with a sample of 834 participants -161 professors and 673 students-, correlations among variables were calculated and a frequencies analysis were performed. Subsequently, a variance analysis was conducted having the moment of the study -beginning or end the course- as the independent variable, as the moderator variable, participant profile -student or professor- and all the mental health variables -depression, anxiety, insomnia, impact of the event Covid-19 and burnout- as dependent variables.

The study findings shows an impact on mental health of students and professor, as a consequence of the Covid-19 pandemic and it is different among students and professors, being the latter the most affected in their mental health by the effects of Covid-19 pandemic.

Keywords: Mental health, depression, pandemic, Covid-19. 


\section{Introducción}

A fines de diciembre de 2019 la comisión municipal de salud de Wuhan -provincia de Hubei, China-, notificó de un conglomerado de casos de neumonía en la ciudad. Posteriormente se confirmó que estos cuadros estaban causados por un nuevo coronavirus, tipificado como Sars-Cov-2, que provoca la enfermedad Covid-19. El 11 de marzo el director general de la Organización Mundial de la Salud -OMS- declaró a esta nueva enfermedad como una pandemia (OMS, 2020).

El surgimiento del nuevo virus Sars-Cov-2, le generó a la comunidad científica total incertidumbre sobre su origen, comportamiento, nivel de letalidad y protocolos de tratamiento médico para la enfermedad Covid-19. Este nivel de incertidumbre la da un perfil completamente distinto a lo que se ha enfrentado con otras pandemias y con ello surge la necesidad del empleo de estrategias de contención agresivas para abordar la crisis sanitaria (OMS, 2020; Taylor, 2019). Una de las estrategias utilizadas por los gobiernos para afrontar la crisis sanitaria por Covid-19, ha sido el distanciamiento social, cuya intención es la de reducir el número de infectados por el virus Sars-Cov-2 y con ello evitar la saturación de la capacidad instalada de los hospitales, dando como resultado la posibilidad de atender a un mayor número de pacientes y reducir el número de muertes ocasionadas por falta de atención médica (Jakovljevic et al., 2020; OMS, 2020).

La estrategia de confinamiento contiene la expansión acelerada de la enfermedad, pero tiene implicaciones importantes en otros aspectos como son: la economía -recursos económicos personales, desempleo o posibilidad de desempleo-, vida social -movilidad, convivencia, interacción-, educación -forma en que se accede a la educación, eliminando la educación presencial y sustituyéndola por educación a distancia-, entre otros aspectos que pueden afectar a la salud mental de las personas (Cullen, Gulati y Kelly, 2020; Levy, 2020). La contención de la expansión del Covid-19 genera dos frentes de batalla, uno sobre la enfermedad y otro relacionado con los efectos colaterales de las medidas tomadas por el confinamiento social que afectan a la economía, vida social, educación y salud mental (Levy, 2020).

La OMS indica que la amenaza a la seguridad y al funcionamiento normal del individuo y de su comunidad que tienen como origen una crisis como la de una pandemia, repercute en más del $80 \%$ de las personas, con un incremento en síntomas que podrían afectar su salud mental manifestándose como angustia, miedo e incluso pánico y terror (Ho, Cheen y Ho, 2020; OMS, 2020). De hecho, se ha documentado qué durante cualquier brote de una enfermedad infecciosa, en la población hay reacciones psicológicas como ansiedad, angustia, depresión y problemas del sueño (Da Silva et al., 2019; Duan y Zhu, 2020; Taylor, 2019).

Esta alteración de la salud mental puede presentarse tanto en personas que se encuentran confinadas, laborando o no, y en personas que se encuentra trabajando, debido a que sus actividades son consideradas como indispensables (Li et al., 2020b; Taylor, 2019). Una evidencia de la afectación a la salud mental de las personas puede apreciarse claramente en la pandemia actual de Covid-19, ya que, en un estudio de 1,210 médicos y enfermeras de 194 ciudades en China, realizado durante enero y febrero de 2020, se encontró que el 54\% de las personas tuvieron un impacto psicológico de moderado a severo; $29 \%$ reportó síntomas de ansiedad de moderada a severa; y 17\% reportó síntomas de depresión moderada a severa 
(Wang et al., 2020). Esta situación pone en perspectiva la afectación de la crisis sanitaria en un grupo que está inmerso en actividades de salud atendiendo esta contingencia.

Es claro, por lo comentado anteriormente, que hay una afectación directa en la salud mental de las personas que se encuentran atendiendo sus actividades laborales, consideradas como esenciales durante la pandemia. Sin embargo, aquellas personas que están en aislamiento social y que atienden actividades a distancia, sin poder salir y con incertidumbre de lo que sucederá con ellos en esta pandemia, también es posible que sean afectadas y es ahí, en donde surge el interés de hacer una investigación sobre cómo se ve impactada la salud mental de personas que desarrollan sus actividades en aislamiento social y en particular, en un sector que no cesó sus actividades, sino que tuvo que adaptarse para continuar operando casi de manera inmediata, este es el caso de algunas universidades privadas en México, que tomaron la decisión de mantener la continuidad de sus cursos mediante la modalidad de educación a distancia.

Por lo que se justifica hacer un estudio para entender el impacto que ha tenido el confinamiento social y el cambio en el sistema de atención de clases presenciales, a clases a distancia, sobre la salud mental de estudiantes y académicos, ubicados en escuelas de negocios, en dos de las universidades privadas líderes en México y que continúan con sus actividades durante la pandemia Covid-19.

\section{Marco teórico}

La agencia de Centros de Control y Prevención de Enfermedades (CDC) perteneciente al gobierno de los Estados Unidos de América y por su parte la OMS, indican conforme a las experiencias obtenidas en las pandemias de VIH, SARS y H1N1, que los brotes epidémicos en general pueden ser estresantes y en particular, el más reciente brote de la enfermedad de Covid-19, puede resultarle estresante a algunas personas, produciendo en ellas temor, ansiedad, preocupación por la salud y la de sus seres queridos, cambios en sus patrones de sueño o alimentación, dificultades para dormir o concentrarse, agravamiento de problemas de salud mental y mayor consumo de alcohol u otras drogas (CDC, 2020; OMS, 2020).

La CDC indica que las personas que pueden tener una reacción más fuerte al estrés de una crisis sanitaria como la del Covid-19, incluyen: adultos mayores y personas con enfermedades crónicas que corren mayor riesgo de enfermarse gravemente por la enfermedad Covid-19, niños y adolescentes. Además de personas que están colaborando en áreas que dan respuesta de emergencia a la pandemia de Covid-19, como lo son; médicos, enfermeras, camilleros, trabajadores sociales, personal de respuesta a emergencias, personas con enfermedades mentales, incluidas las personas con trastornos por abuso de sustancias (CDC, 2020). La OMS indicó desde que se enfrentó la pandemia de la Influenza H1N1, que este tipo de crisis sanitaria provoca un efecto negativo sobre las personas en su salud mental, estilo de vida y economía, ocasionado por los efectos traumáticos, emocionales y afectivos que son causados por la enfermedad subyacente a la pandemia y por el aislamiento social que se ejerce para contener los brotes epidémicos (OMS, 2005).

Hay cuatro periodos pandémicos claramente identificados: el periodo inter pandémico, en el que no se ha detectado ningún virus en seres humanos y que puede llegar hasta la detección de virus en animales e identificar riesgos para la salud humana. La alerta pandémica, que se produce cuando hay uno o varios casos detectados en humanos que se expande a conglomerados pequeños con propagación bien localiza- 
da, en los que el virus se adapta bien a los seres humanos, expandiéndose a grupos cada vez más grandes. El periodo pandémico, en el que la transmisión se propaga de forma intensa y continuada en la población en general causando una crisis sanitaria y finalmente el periodo post pandémico, en el que se regresa al periodo inter pandémico (OMS, 2005).

La salud mental de los individuos que viven la pandemia se ve comprometida de manera distinta en cada fase de la pandemia y por ello resulta importante identificar la forma en que esta se ve afectada en las diferentes etapas del proceso pandémico (OMS, 2020).

Hay diversas investigaciones que han estudiado el efecto de las pandemias sobre la salud mental, como los resultados de una investigación realizada en 2009, en la pandemia de la Influenza H1N1, en la que se estudió el impacto que tuvo la incertidumbre que manifestaron los pacientes con antecedentes de enfermedades mentales de larga duración como la depresión, sobre verse contagiados en el periodo pandémico (Page et al., 2011). En el estudio se encontró una afectación que fue de moderada a severa en su nivel de preocupación por la posibilidad de infectarse de Influenza H1N1 y como consecuencia un deterioro de su salud mental, por el incremento de ansiedad, tristeza, pensamientos negativos y problemas para conciliar el sueño (Page et al., 2011). Por otra parte, en un estudio realizado en China, en Hubei y fuera de Hubei, durante el periodo pandémico de la crisis de Covid-19, se evaluó el impacto sobre la salud mental de 1,257 médicos y enfermeras durante la atención de la crisis sanitaria, la investigación mostró una afectación importante en síntomas de depresión en un 50\% de ellos, insomnio en el 34\% y angustia en el 72\% (Li et al. 2020a).

Adicionalmente, en un meta estudio que analizó el impacto de los brotes de epidemia viral en la salud mental de los trabajadores de salud, en el que recopilaron los datos de 61 estudios realizados durante la epidemia de SARS, se encontró que la afectación sobre la salud mental fue mayor para la ansiedad con el 45\% de los casos, seguida de la depresión con 38\%, el trastorno de estrés agudo con 31\%, agotamiento con $29 \%$ y trastorno de estrés postraumático 19\% (Ricci-Cabello et al, 2020). Con base en las investigaciones mencionadas y que fueron realizadas a nivel internacional, en una crisis sanitaria, en la fase pandémica, tanto para pacientes como para médicos y enfermeras. Se aprecia que hay una afectación a la salud mental de estos individuos que va desde moderada hasta severa, presentando síntomas de depresión, ansiedad, angustia, insomnio, agotamiento y estrés agudo.

En México, en la fase pandémica, la Universidad Iberoamericana realizó una encuesta telefónica representativa de la población mexicana para dar seguimiento a los efectos del Covid-19 en el bienestar de los hogares mexicanos conocida como: ENCOVID19 (2020). En la encuesta se tuvieron los siguientes hallazgos: se estimó que entre 5.2 y 8.1 millones de personas fueron descansadas o no pudieron salir en busca de trabajo en el contexto de la pandemia del Covid-19, se estima que uno de cada tres hogares, observó una reducción del 50\% de su ingreso entre febrero y marzo 2020 e indica que el 64\% de la población declaraba estar cumpliendo con la instrucción de confinamiento en el domicilio y que el 46\% contaba con recursos para mantenerse hasta el 30 de abril de 2020. Además de que el 46\% de la población mayor de 18 años cree muy probable o probable que se infecte de Covid-19, que el 53\% de la población cree que si alguien se infecta puede ser muy peligroso, que el 50\% de la población tiene una preocupación muy alta de que su salud se vea afectada por el coronavirus y finalmente el $27 \%$ de los encuestados mayores de 18 años manifestaron síntomas depresivos y severos de ansiedad (ENCOVID19, 2020) 
En el estudio hecho por la Universidad Iberoamericana en México en la fase pandémica, se observa que al igual que lo que pasa a nivel internacional, hay impactos sobre la salud mental, ya que se encontraron síntomas importantes de depresión y de ansiedad (ENCOVID19, 2020). Esto permite asumir que la pandemia del Covid-19 afectará la salud mental de la población en México en lo general. Sin embargo, no hay información en los estudios revisados de cómo evolucionará esta afectación a lo largo de las diferentes fases pandémicas, lo cual permite abrir una línea de investigación sobre este aspecto.

En el ámbito de la salud pública, en México se implementaron medidas de higiene como lavarse las manos y distanciamiento social como mecanismos primordiales para controlar la expansión acelerada de la pandemia del Covid-19. El cierre de escuelas fue la primera medida distanciamiento social que instauró el gobierno en el país, siendo efectiva desde el 20 de marzo de 2020 (Secretaría de Salud, 2020).

Las escuelas cerraron las aulas físicamente, aunque algunas instituciones de educación superior dieron continuidad a sus cursos de manera casi inmediata -entre estas el Tecnológico de Monterrey, las Universidades Anáhuac e Iberoamericana-, mediante la educación en modalidad a distancia, dando seguimiento a su proceso educativo, en el que estuvieron involucrados alumnos y profesores. Las clases se efectuaron con ayuda de las tecnologías de información y con ello culminaron sus cursos académicos del primer semestre de 2020 y de hecho continúan con sus programas a distancia hasta que sea posible retomar la asistencia a las aulas.

La educación a distancia fue la alternativa que a nivel internacional tomaron los colegios y universidades para mantener la continuidad de sus programas en cerca de 200 países y en la que alrededor de 1,600 millones de niños, adolescentes y jóvenes se han visto involucrados (UNESCO, 2020, IESALC-UNESCO, 2020). Esta modalidad fue denominada por Hodges y colaboradores como "enseñanza remota de emergencia" (Hodges et al., 2020), ya que, a diferencia de programas de educación a distancia bien diseñados y planificados, las instituciones educativas, profesores y alumnos tuvieron que resolver en una situación de emergencia como mantener la continuidad de la educación (García, 2020).

Los programas formales de educación a distancia que han sido diseñados con este objetivo difieren grandemente de los de enseñanza remota de emergencia, ya que los primeros cuentan con una tecnología educativa robusta y planificada, mientras que los segundos han sido improvisados para mantener la continuidad educativa en una situación de emergencia y con ello acarrean varios problemas entre los cuales están los metodológicos, infraestructura inapropiada -computadoras, comunicación, software-, falta de capacitación de los docentes y alumnos en el uso del software y tecnologías educativas a distancia, habilidades de trabajo, entre otros (García, 2020; Hodges et al., 2020). Causando con ello un incremento repentino de trabajo y estrés a los profesores y alumnos, frustración por fallas tecnológicas y uso de técnicas didácticas improvisadas, además de la sensación de despersonalización en las clases y lidiar con el aislamiento social (García, 2020, Hodges et al., 2020; Means, Bakiam y Murphy, 2014).

Ante el inicio convencional de clases y unos meses después la declaración de la pandemia que provocaron el cambio de clases presenciales a una enseñanza remota de emergencia ha sido un momento único para realizar un estudio sobre los efectos de la pandemia en la salud mental en estudiantes y profesores, por lo que aprovechamos estas circunstancias para investigar dicha afectación en el periodo inter pandémico y pandémico, para lo cual se propusieron las siguientes hipótesis: 
- H1: Hay una relación positiva entre las variables depresión, ansiedad, impacto del evento, insomnio y agotamiento.

- H2: Hay evidencia de afectaciones a la salud mental de alumnos y profesores como consecuencia de la pandemia Covid-19 en aspectos como: depresión, ansiedad, impacto del evento, insomnio y agotamiento.

- H3: Hay una afectación diferenciada en la salud mental de alumnos y profesores como consecuencia de la pandemia Covid-19.

\section{Materiales y métodos}

Este estudio es no experimental, ex post facto, longitudinal de carácter descriptivo, realizado en el periodo inter pandémico -inicio de cursos en enero y febrero de 2020 y pandémico -fin de cursos en junio de 2020-.

\subsection{Muestra}

En esta investigación se seleccionaron a estudiantes y profesores de escuelas de negocios de universidades privadas mexicanas ubicadas en los tres primeros lugares en México de acuerdo con la clasificación de QS World University Ranking 2019. La muestra fue por conveniencia, en la que se entrevistó a estudiantes y profesores que estaban en sus cursos en el primer semestre de 2020, por lo que durante el semestre asistieron a sus clases presenciales desde enero, antes de la declaración de la pandemia y concluyeron sus cursos en la modalidad a distancia -enseñanza remota de emergencia- en el mes de junio. Participaron 673 alumnos y 161 profesores de las universidades seleccionadas.

La muestra de estudiantes estuvo integrada por 316 mujeres y 389 hombres y para los profesores 98 mujeres y 63 hombres. El rango de edad de los estudiantes fue de 19 a 24 años -Media $(\mathrm{M})=21.6$ años, Desviación estándar $(\mathrm{DE})=1.5$ y para los profesores el rango de edad fue de 28 a 55 años $-\mathrm{M}=37.6$ años, $\mathrm{DE}=18.5$-. El número de materias a tomar por alumno en promedio fueron 5.6 $-\mathrm{M}=5.6, \mathrm{DE}=1.8-\mathrm{y}$ las materias impartidas por profesor en promedio fueron $3.3-\mathrm{M}=3.3$; $\mathrm{DE}=1.2-$.

\subsection{Aplicación del cuestionario}

Se diseñó un cuestionario en el software "survey mokey" que estuvo integrado por las secciones: datos generales, síntomas de depresión, ansiedad, angustia, insomnio y burnout. Para realizar el estudio la decisión fue tomar una muestra por conveniencia, en la que se identificaron dos grupos para aplicar el cuestionario: el primer grupo estuvo integrado por alumnos que cursaban los cursos del primer semestre de 2020 y otro de profesores que impartían clase en el citado semestre. Se le hizo una invitación a participar en una investigación sobre el impacto de la pandemia de Covid-19 -en un principio epidemia y después pandemia- sobre su estado de ánimo - . Indicando que la información sería empleada para fines de estudios académicos y cuyos datos se tratarían en conjunto con los de todos los participantes y que se garantizaría con ello la confidencialidad de sus respuestas individuales. 
La invitación a participar se hizo por correo electrónico, en el cual se anexó la liga para acceder al cuestionario -se enviaron 900 invitaciones a alumnos y 250 a profesores- y se les dio un plazo de 2 semanas para contestar, enviando al fin de la primera semana un recordatorio. Al final la efectividad de respuesta fue de 74\% para alumnos -673 cuestionarios útiles- y 65\% para profesores, con 673 y 161 cuestionarios útiles respectivamente.

\subsection{Instrumentos de medición}

\subsubsection{Síntomas de depresión}

El cuestionario PHQ-9 -Patient Health Questionare- es uno de los instrumentos más usados en el mundo para diagnosticar la depresión, además que permite identificar el grado de afectación que sufre el individuo y consta de nueve ítems. En este estudio se empleó la versión en español validada para su uso por Baader et al., (2012). En el instrumento se le pide al participante indicar con qué frecuencia le han afectado algunos de los siguientes problemas durante las últimas dos semanas. Un ejemplo de los ítems es el siguiente: "se ha sentido mal consigo mismo, ha sentido que usted es un fracaso o ha sentido que se ha fallado a sí mismo o a su familia". Las respuestas se dan en una escala de frecuencia subjetiva de cinco puntos que van de 0 "nunca" a 4 "casi todos los días". Mayores puntuaciones muestran mayores niveles de depresión. La consistencia interna fue medida para este estudio con un coeficiente de alfa de Cronbach de 0.89 .

\subsubsection{Ansiedad}

La escala del trastorno de ansiedad generalizada (GAD-7) (Spitzer et al., 2006), es un instrumento empleado para el diagnóstico de la ansiedad y permite identificar el nivel de afectación en los individuos. La escala validada se encuentra disponible en español. El instrumento consta de siete ítems y en él se le pide al participante indicar con qué frecuencia le han afectado algunos de los siguientes problemas durante las últimas dos semanas. Un ejemplo de los ítems es el siguiente: "se siente asustado como si algo horrible pudiese pasar". Las respuestas se dan en una escala de frecuencia subjetiva de cinco puntos que van de 0 "nunca" a 4 "casi todos los días". Mayores puntuaciones muestran mayores niveles de ansiedad. La consistencia interna fue medida para este estudio con un coeficiente de alfa de Cronbach de 0.93.

\subsubsection{Impacto del evento}

La escala del impacto del evento revisada (IES-R) (Weiss y Marmar, 1997) se emplea para medir el nivel de estrés ocasionado por un evento sobre el individuo. Esta escala consta de veintidós ítems y evalúa tres constructos: intrusión, evitación e hiperactivación, ante la presencia de un acontecimiento traumático. En el instrumento se le pide que lea cada frase del cuestionario e indique cuál es su sentir de molestia sobre cada uno de los ítems revisados, esto en la última semana. Un ejemplo de los ítems es el siguiente: "sentía como si esto no hubiese sucedido o no fuese real". Las respuestas se dan en una escala de frecuencia subjetiva de cinco puntos que van de 0 "nada" a 4 "extremadamente ". Mayores puntuaciones muestran mayores niveles de impacto. 
La consistencia interna fue medida para este estudio con un coeficiente de alfa de Cronbach de 0.88 y el análisis factorial ejecutado mediante el método de máxima verosimilitud con rotación varimax, aportó tres factores: intrusión, evitación e hiperactivación. Estos factores explicaron el 73\% de la varianza. La prueba de factor único generó una varianza común del 15\% -si hubiera algún problema de varianza asociada al método, el factor debería explicar el 50\% o más de la varianza como indican Podsakoff et al. (2003). Los resultados del análisis factorial confirmatorio del instrumento IES-R para este estudio son: $\mathrm{X}^{2}=1789.25 ; \mathrm{CFI}=0.91 ; \mathrm{TLI}=0.90 ; \mathrm{AIC}=82856.61 ; \mathrm{RMSEA}=0.04, \mathrm{SRMR}=0.05$ y muestran buenos parámetros de ajuste de acuerdo con los parámetros especificados por Hu y Bentler, 1999 y Pérez, 2004.

\subsubsection{Síntomas de insomnio}

La escala de índice de severidad del insomnio (ISI) (Bastien, Valliéres y Morin, 2001) se emplea para medir el nivel afectación por insomnio sobre el individuo. Esta escala consta de siete ítems y evalúa aspectos relacionados con la dificultad para dormir y el insomnio. Esta escala se encuentra disponible en español. En el instrumento se le pide al participante que lea cada frase del cuestionario e indique cuál es su sentir sobre cada uno de los ítems revisado, esto en las últimas dos semanas. Un ejemplo de los ítems es el siguiente: "tengo dificultad para conciliar el sueño". Las respuestas se dan en una escala de frecuencia subjetiva de cinco puntos que van de 0 "nada" a 4 "extremadamente ". Mayores puntuaciones muestran mayores niveles de impacto. La consistencia interna fue medida para este estudio con un coeficiente de alfa de Cronbach de 0.88 .

\subsubsection{Agotamiento -Burnout-}

El agotamiento o burnout, se midió con la escala de Maslach y Jackson (1981), en su versión en español y que consta de 22 ítems. Los entrevistados fueron cuestionados con relación a cada afirmación del cuestionario, a la cual debían contestar empleando una escala de frecuencia subjetiva que va de " 0 " nunca a "6" todos los días. La escala fue validad para su uso en México por Colín (2019). En esta escala, se evalúan tres dimensiones: desgaste emocional, despersonalización y reducción de la eficacia personal. A continuación, se muestra ejemplos de los ítems para cada factor. Desgaste emocional con nueve ítems, por ejemplo: "me siento emocionalmente agotado por mi trabajo". En el caso de despersonalización la escala está compuesta por cinco ítems, por ejemplo: "siento que me he hecho más duro al tratar a los colaboradores". La escala de la eficacia personal está integrada por ocho ítems, por ejemplo: "siento que puedo entender fácilmente a los colaboradores".

La consistencia interna para este estudio medida con el coeficiente alfa de Cronbach fue: burnout, 0.85; desgaste emocional, 0.87 ; despersonalización, 0.82 y reducción de la eficacia personal, 0.88 y el análisis factorial realizado mediante el método de máxima verosimilitud con rotación varimax, arrojó tres factores: desgaste personal, despersonalización y reducción de la eficacia personal. Los factores explican el $75 \%$ de la varianza. La prueba de factor único mostró una varianza común del 15\%. Los resultados del análisis factorial confirmatorio del instrumento de agotamiento para este estudio son: $\mathrm{X}^{2}=$ 2459.52; $\mathrm{CFI}=0.91 ; \mathrm{TLI}=0.91 ; \mathrm{RMSEA}=0.05, \mathrm{SRMR}=0.04$ y muestran buenos parámetros de ajuste de acuerdo con los parámetros especificados por Hu y Bentler, 1999 y Pérez, 2004 


\subsection{Análisis de datos}

La información se recopiló en dos momentos: al inicio del curso en febrero de 2020, situado antes de la declaración de la pandemia y al fin del curso en junio de 2020, con la pandemia ya declarada y que coincide con un incremento importante de casos de Covid-19 en México -al 29 de junio de 2020, se registraron 220,657 casos confirmados de Covid-19 y un total de 27,121 muertes asociadas con la enfermedad- (Secretaría de Salud, 2020).

Se realizó el análisis descriptivo de los datos obtenidos en las encuestas para cada variable: depresión, ansiedad, impacto del evento, problemas del sueño y agotamiento.

Se analizó la correlación entre las variables en los dos momentos en que se efectuó el estudio y para ello se empleó el coeficiente de correlación de Spearman.

Para establecer la clasificación sobre la intensidad de la afectación a la salud mental de los participantes, se empleó el punto de corte recomendado por los autores de cada escala, que se ubica desde leve hasta severa. Con estos criterios se realizó un análisis de frecuencia para los dos momentos del estudio, en el que se pudo identificar la variación en la intensidad de la afectación para los participantes. Para la hipótesis relativa sobre el impacto diferenciado entre estudiantes y profesores, se empleó el análisis de varianza con medidas repetidas para evaluar las variables dependientes para el inicio y final del curso.

\section{Resultados}

Como ha sido mencionado, el estudio presenta dos momentos: el inicio del curso en febrero -previo a la declaración de la pandemia- y el final del curso en junio de 2020 -ya declarada la pandemia, con un avance importante en los contagios, decesos, varios días de confinamiento y afectaciones económicas-, por lo que se muestran los resultados de estos dos momentos para su análisis y seguimiento. En la tabla 1, se aprecia la evaluación hecha por los estudiantes y profesores al inicio del curso, sobre las diferentes variables de salud mental y en los resultados se aprecian indicadores bajos en lo general. Por otra parte, se identifican correlaciones de moderadas a altas entre las variables depresión, ansiedad, insomnio y agotamiento. En cuanto a la correlación entre impacto del evento -en este caso Covid-19- la correlación de esta variable con las demás se observa como débil. 


\section{Tabla 1}

Estadísticos descriptivos, correlaciones bivariadas de las variables estudiadas $(n=834)$ - inicio del curso (febrero 2020)

\begin{tabular}{|c|c|c|c|c|c|c|c|}
\hline & $\begin{array}{l}\text { Promedio } \\
\text { (M) }\end{array}$ & $\begin{array}{l}\text { Desviación } \\
\text { Estándar } \\
\text { (DE) }\end{array}$ & 1 & 2 & 3 & 4 & 5 \\
\hline 1. Depresión $(P H Q-9)$ & 1.1 & 0.1 & 1 & & & & \\
\hline 2. Ansiedad (GAD-7) & 1.2 & 0.3 & $0.58 * *$ & 1 & & & \\
\hline 5. Insomnio (SIS) & 1.5 & 0.2 & $0.64 * *$ & $0.53 * *$ & 1 & & \\
\hline 4. Impacto del evento (IES-R) & 1.2 & 0.1 & $0.23 * *$ & $0.17 * *$ & $0.08 *$ & 1 & \\
\hline 5. Agotamiento (Burnout) & 2.1 & 0.3 & $0.66^{k * *}$ & $0.58 * *$ & $0.71 * *$ & $0.12 *$ & 1 \\
\hline
\end{tabular}

En la tabla 2, se observa la evaluación hecha por los estudiantes y profesores al final de curso, sobre las diferentes variables de salud mental y en los resultados muestran un cambio radical sobre los registrados en febrero 2020, estos incrementaron su intensidad casi al doble. Asimismo, las correlaciones pasaron a ser altas entre las variables depresión, ansiedad, insomnio y agotamiento. En particular para la variable impacto del evento -en este caso Covid-19- la correlación de esta variable pasó a ser de moderada a alta.

\section{Tabla 2}

Estadísticos descriptivos, correlaciones bivariadas de las variables estudiadas $(n=834)-$ fin del curso (junio 2020)

\begin{tabular}{|c|c|c|c|c|c|c|c|}
\hline & $\begin{array}{l}\text { Promedio } \\
\text { (M) }\end{array}$ & $\begin{array}{l}\text { Desviación } \\
\text { Estándar } \\
\text { (DE) }\end{array}$ & 1 & 2 & 3 & 4 & 5 \\
\hline 1. Depresión (PHQ-9) & 2.7 & 0.2 & 1 & & & & \\
\hline 2. Ansiedad $(G A D-7)$ & 2.9 & 0.3 & $0.63 * *$ & 1 & & & \\
\hline 5. Insomnio (SIS) & 2.1 & 0.2 & $0.71 * *$ & $0.68 * *$ & 1 & & \\
\hline 4. Impacto del evento (IES-R) & 3.1 & 0.3 & $0.56 * *$ & $0.61 * *$ & $0.56 * *$ & 1 & \\
\hline 5. Agotamiento (Burnout) & 4.8 & 0.3 & $0.81^{k * *}$ & $0.72 * *$ & $0.83 * *$ & $0.61 * *$ & 1 \\
\hline
\end{tabular}

Los datos que se encuentran registrados sobre el nivel de salud mental de los estudiantes y profesores (ver tabla 3 y tabla 4), representados por las variables depresión, ansiedad, insomnio, impacto sobre el evento y agotamiento, registran un cambio importante en su intensidad desde el inicio de la pandemia, que coincide con el inicio de los cursos, hasta el final de los cursos, momento en que la pandemia se encuentra en plena expansión en México. En el inicio de los cursos, la evaluación del nivel de la salud mental de los estudiantes estaba a niveles normales o leves y al avanzar la pandemia, justo al finalizar los cursos, los niveles de las variables registraron una mayor afectación en los niveles de moderado y severo. 
En el caso de la depresión, al inicio del curso el porcentaje de personas que declararon niveles normales y leves estaban en un $91.5 \%$-nivel entre moderado y severo de $8.5 \%$-, mientras que al fin del curso los niveles entre moderado y severo se ubicó en un $28.3 \%$. En relación con la ansiedad, en febrero los niveles normales y leves se ubicaban en $89.9 \%$-nivel entre moderado y severo de $10.1 \%$ - y en junio entre moderada y severa se quedó en $28.5 \%$. Por su parte, el insomnio se reportaba al inicio del año a niveles normales y leves en un $90.5 \%$-nivel entre moderado y severo $9.5 \%$ - y a junio entre moderado a alto un $21.2 \%$. El impacto del evento Covid-19 a inicios del año los niveles normales a leves estaban en $96.2 \%$-nivel entre moderado y severo 3.8\% y al fin de los cursos en un nivel de moderado a alto llegó a $29.0 \%$. Por último, el agotamiento percibido al inicio del curso en niveles normales o leves se ubicaba en un $87.3 \%$-nivel entre moderado y severo $12.7 \%$ - y al final del curso el agotamiento de moderado a severo se ubicó en $50.8 \%$.

Tabla 3

Nivel de afectación en las variables de salud mental por tipo de participante al inicio del curso $(n=834)$

\begin{tabular}{|c|c|c|c|c|c|c|c|}
\hline \multirow[b]{2}{*}{ Nivel de afectación } & \multicolumn{7}{|c|}{ Inicio del curso (febrero 2020) } \\
\hline & Total & $\%$ & Estudiantes & $\%$ & Profesores & $\%$ & $p$ Value \\
\hline \multicolumn{8}{|l|}{ Depresión $(P H Q-9)$} \\
\hline Normal & 517 & 62.0 & 441 & 65.5 & 76 & 47.5 & \\
\hline Leve & 246 & 29.5 & 190 & 28.2 & 57 & 35.1 & $0.01<$ \\
\hline Moderado & 44 & 5.2 & 28 & 4.1 & 16 & 10.0 & \\
\hline Severo & 27 & 3.2 & 15 & 2.2 & 12 & 7.4 & \\
\hline \multicolumn{8}{|l|}{ Ansiedad (GAD-7) } \\
\hline Normal & 452 & 54.3 & 376 & 55.8 & 77 & 47.8 & \\
\hline Leve & 297 & 35.6 & 235 & 34.9 & 62 & 38.7 & $0.01<$ \\
\hline Moderado & 65 & 7.7 & 48 & 7.2 & 16 & 10.0 & \\
\hline Severo & 20 & 2.4 & 14 & 2.1 & 6 & 3.5 & \\
\hline \multicolumn{8}{|l|}{ Ins omnio (SIS) } \\
\hline Normal & 616 & 73.9 & 505 & 75.1 & 111 & 68.7 & \\
\hline Leve & 139 & 16.6 & 104 & 15.4 & 35 & 21.8 & $0.01<$ \\
\hline Moderado & 64 & 7.6 & 51 & 7.6 & 13 & 7.8 & \\
\hline Severo & 16 & 1.9 & 13 & 1.9 & 3 & 1.7 & \\
\hline \multicolumn{8}{|c|}{ Impacto del evento (IES-R) } \\
\hline Normal & 731 & 87.7 & 589 & 87.5 & 142 & 88.5 & \\
\hline Leve & 71 & 8.5 & 59 & 8.7 & 13 & 7.8 & $0.05<$ \\
\hline Moderado & 25 & 3.0 & 21 & 3.1 & 4 & 2.4 & \\
\hline Severo & 7 & 0.8 & 5 & 0.7 & 2 & 1.3 & \\
\hline \multicolumn{8}{|c|}{ Agotamiento (Burnout) } \\
\hline Normal & 535 & 64.1 & 445 & 66.1 & 90 & 55.8 & \\
\hline Leve & 194 & 23.2 & 151 & 22.4 & 43 & 26.8 & $0.01<$ \\
\hline Moderado & 87 & 10.4 & 68 & 10.1 & 19 & 11.6 & \\
\hline Severo & 19 & 2.2 & 9 & 1.4 & 9 & 5.8 & \\
\hline
\end{tabular}


En todas las variables hubo un incremento en la percepción de deterioro de la salud mental, siendo la más impactante la del agotamiento. Puede apreciarse que el impacto sobre las diferentes variables de salud mental es menos intenso en los estudiantes. Los niveles de afectación entre moderada y severa en los profesores son mayores a los que registran los estudiantes y en junio, alcanzaron los siguientes valores: depresión, 35.7\%; ansiedad, 49.7\%; insomnio, 43.6\%; impacto del evento, 53.5\% y agotamiento, 51.5\%. Esto implicó un incremento de casi al doble en los profesores con nivel de depresión de moderada a alta y en mayor proporción entre las demás variables, situación que muestra un alto impacto en estos participantes al momento del fin del curso.

Tabla 4

Nivel de afectación en las variables de salud mental por tipo de participante al fin del curso $(n=834)$

\begin{tabular}{|c|c|c|c|c|c|c|c|}
\hline Nivel de afectación & Total & $\%$ & Estudiantes & $\%$ & Profesores & $\%$ & $p$ Value \\
\hline \multicolumn{8}{|l|}{ Depresión $(P H Q-9)$} \\
\hline Normal & 298 & 35.7 & 240 & 35.7 & 58 & 35.8 & \multirow{4}{*}{$0.01<$} \\
\hline Leve & 300 & 36.0 & 254 & 37.8 & 46 & 28.5 & \\
\hline Moderado & 191 & 22.9 & 151 & 22.4 & 40 & 25.1 & \\
\hline Severo & 45 & 5.4 & 28 & 4.1 & 17 & 10.6 & \\
\hline \multicolumn{8}{|l|}{ Ansiedad (GAD-7) } \\
\hline Normal & 328 & 39.3 & 279 & 41.5 & 49 & 30.2 & \multirow{4}{*}{$0.01<$} \\
\hline Leve & 269 & 32.2 & 236 & 35.1 & 32 & 20.1 & \\
\hline Moderado & 175 & 21.0 & 126 & 18.7 & 50 & 30.8 & \\
\hline Severo & 62 & 7.4 & 32 & 4.7 & 30 & 18.9 & \\
\hline \multicolumn{8}{|l|}{ Ins omnio (SIS) } \\
\hline Normal & 480 & 57.5 & 418 & 62.1 & 62 & 38.5 & \multirow{4}{*}{$0.01<$} \\
\hline Leve & 178 & 21.3 & 149 & 22.1 & 29 & 17.9 & \\
\hline Moderado & 133 & 16.0 & 81 & 12.1 & 52 & 32.1 & \\
\hline Severo & 43 & 5.2 & 25 & 3.7 & 19 & 11.5 & \\
\hline \multicolumn{8}{|c|}{ Impacto del evento (IES-R) } \\
\hline Normal & 358 & 43.0 & 308 & 45.8 & 50 & 31.1 & \multirow{4}{*}{$0.05<$} \\
\hline Leve & 235 & 28.1 & 210 & 31.2 & 25 & 15.4 & \\
\hline Moderado & 182 & 21.8 & 115 & 17.1 & 67 & 41.5 & \\
\hline Severo & 59 & 7.1 & 40 & 5.9 & 19 & 12.0 & \\
\hline \multicolumn{8}{|c|}{ Agotamiento (Burnout) } \\
\hline Normal & 292 & 35.1 & 246 & 36.5 & 47 & 29.1 & \multirow{4}{*}{$0.01<$} \\
\hline Leve & 140 & 16.7 & 108 & 16.1 & 31 & 19.4 & \\
\hline Moderado & 258 & 31.0 & 194 & 28.9 & 64 & 39.7 & \\
\hline Severo & 144 & 17.2 & 125 & 18.5 & 19 & 11.8 & \\
\hline
\end{tabular}

Ante el comportamiento que mostraron los dos grupos de estudio: estudiantes y profesores, se realizó un análisis de varianza para identificar si hay una diferencia en el impacto en la salud mental de los estudiantes o profesores ante el avance de la pandemia Covid-19. En el análisis de varianza se tomó como 
variable independiente (VI) al momento del curso que coincide con el estatus de la pandemia -inicio y fin de cursos-, la variable dependiente (VD) para cada caso, las variables de salud mental evaluada -depresión, ansiedad, insomnio, impacto del evento y agotamiento-, la variable moderadora (VM) fue el perfil del participante: estudiante o profesor. Los resultados del análisis de varianza se muestran en la tabla 5.

Los datos muestran que hay una relación estadísticamente significativa entre la variable moderadora y la variable independiente para cada variable dependiente evaluada. Lo que implica que hay un comportamiento distinto entre los alumnos y profesores frente a la pandemia -inicio de cursos, pandemia no declarada y en fin de cursos, pandemia en expansión- y quienes ha tenido mayor afectación han sido los profesores.

\section{Tabla 5}

Aova entre la VI= momentos de curso: inicio y fin del curso, VD y VM (moderadora)= perfil del participante $(n=834)$

\begin{tabular}{|c|c|c|c|c|c|}
\hline \multicolumn{6}{|c|}{ Anova entre la VI= momentos del curso: inicio y fin del curso, VD y VM (moderadora)= perfil del participante $(\mathrm{N}=834)$} \\
\hline \multicolumn{6}{|c|}{ VD: Nivel de depresión } \\
\hline Fuente de variación & $\mathrm{SC}$ & $\mathrm{gl}$ & $\mathrm{MC}$ & $\mathrm{F}(0.01)$ & $P$ \\
\hline VI: Momento del curso (inicio y fin) & 133.1 & 1 & 103.1 & 1449.2 & 0.000 \\
\hline Interacción: Momento del curso*Perfil del participante & 105.9 & 1 & 105.9 & 1153 & 0.000 \\
\hline Error & 76.4 & 832 & 0.1 & & \\
\hline \multicolumn{6}{|l|}{ VD: Nivel de ansiedad } \\
\hline Fuente de variación & $\mathrm{SC}$ & $\mathrm{gl}$ & $\mathrm{MC}$ & $\mathrm{F}(0.01)$ & $P$ \\
\hline VI: Momento del curso (inicio y fin) & 100.6 & 1 & 100.6 & 981.1 & 0.000 \\
\hline Interacción: Momento del curso*Perfil del participante & 168.9 & 1 & 168.9 & 1648.3 & 0.000 \\
\hline Error & 85.3 & 832 & 0.1 & & \\
\hline \multicolumn{6}{|l|}{ VD: Nivel de insomnio } \\
\hline Fuente de variación & $\mathrm{SC}$ & $\mathrm{gl}$ & $\mathrm{MC}$ & $\mathrm{F}(0.01)$ & $P$ \\
\hline VI: Momento del curso (inicio y fin) & 84.2 & 1 & 81.2 & 614.9 & 0.000 \\
\hline Interacción: Nivel de insomnio * perfil del participante & 31.5 & 1 & 31.5 & 238.3 & 0.000 \\
\hline Error & 109.9 & 832 & 0.1 & & \\
\hline \multicolumn{6}{|l|}{ VD: Nivel de impacto del evento } \\
\hline Fuente de variación & SC & $\mathrm{gl}$ & $\mathrm{MC}$ & $\mathrm{F}(0.01)$ & $P$ \\
\hline VI: Momento del curso (inicio y fin) & 219.5 & 1 & 219.5 & 824.0 & 0.000 \\
\hline Interacción: Nivel de insomnio * perfil del participante & 16.9 & 1 & 16.9 & 63.4 & 0.000 \\
\hline Error & 221.6 & 832 & 0.3 & & \\
\hline \multicolumn{6}{|l|}{ VD: Nivel de agotamiento } \\
\hline Fuente de variación & $\mathrm{SC}$ & $\mathrm{gl}$ & $\mathrm{MC}$ & $\mathrm{F}(0.01)$ & $P$ \\
\hline VI: Momento del curso (inicio y fin) & 119.3 & 1 & 119.4 & 237.7 & 0.000 \\
\hline Interacción: Nivel de insomnio * perfil del participante & 67.5 & 1 & 67.5 & 134.6 & 0.000 \\
\hline Error & 417.4 & 832 & 0.5 & & \\
\hline
\end{tabular}

Sc: Suma de cuadrados, MC:: media cuadrática 
En las fíguras de la 1 a la 5 se puede apreciar la interacción entre estudiantes y profesores, en las que se observa en los estudiantes un menor nivel de afectación -número menor en los indicadores de salud mental, lo que implica mejor salud mental- y en el caso de los profesores, un mayor cambio que el presentado por los estudiantes ante la pandemia.

Hubo un fenómeno particular en el caso del impacto del evento Covid-19, que es en la única variable que el comportamiento entre estudiantes y profesores muestran una mayor simetría (ver Figura 4).

Por otra parte, hay otra situación que llama la atención, en relación con la variable agotamiento, los estudiantes muestran un incremento muy importante al final del curso, comparativamente con lo registrado por los profesores, lo que muestra que es la variable que presenta mayor impacto sobre este grupo de estudio (ver Figura 5).

\section{Figura 1}

Análisis de moderación: perfil del participante sobre el momento del curso y la variable depresión $(n=834)$

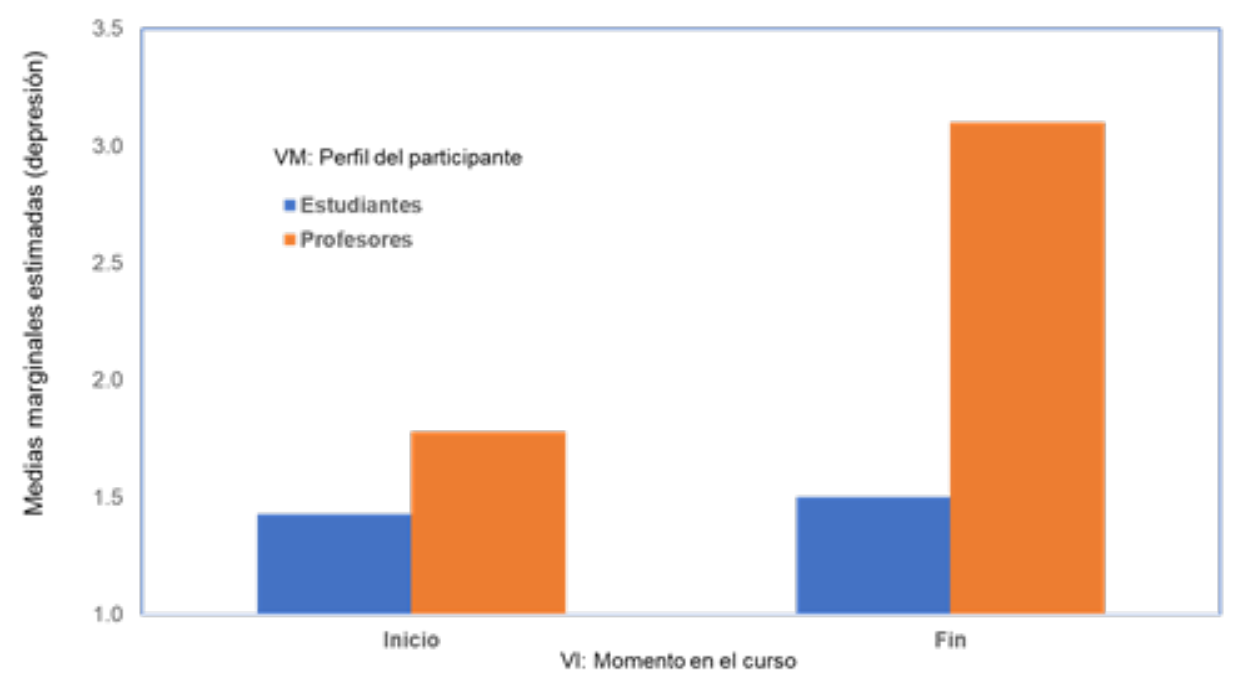


Colín Flores, C. G.

Figura 2

Análisis de moderación: perfil del participante sobre el momento del curso y la variable ansiedad $(n=834)$

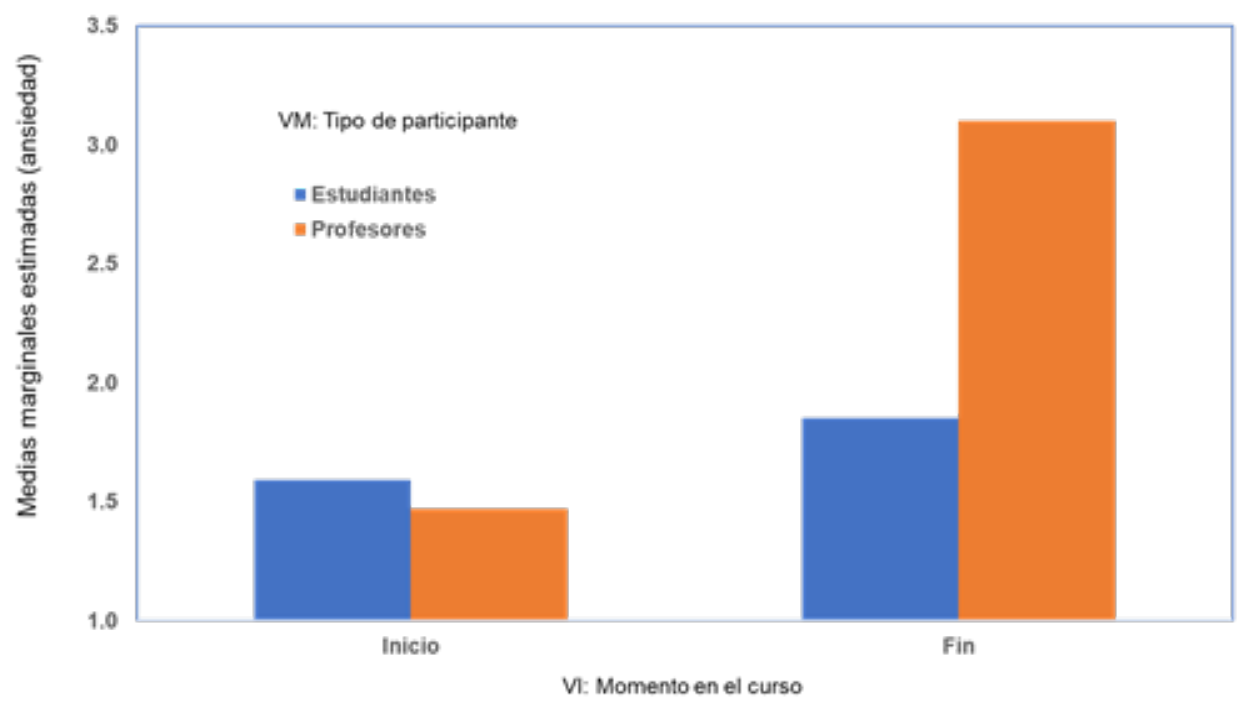

Figura 3

Análisis de moderación: perfil del participante sobre el momento del curso y la variable insomnio $(n=834)$

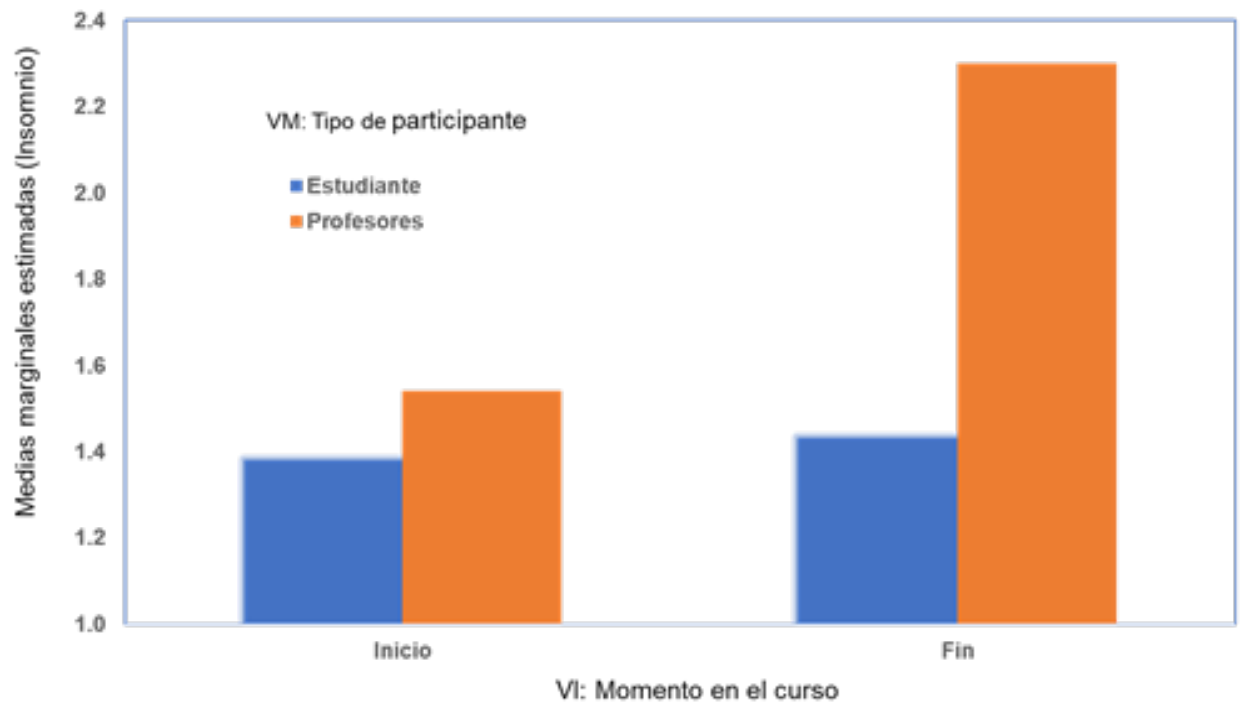




\section{Figura 4}

Análisis de moderación: perfil del participante sobre el momento del curso y la variable impacto del evento ( $n=834)$

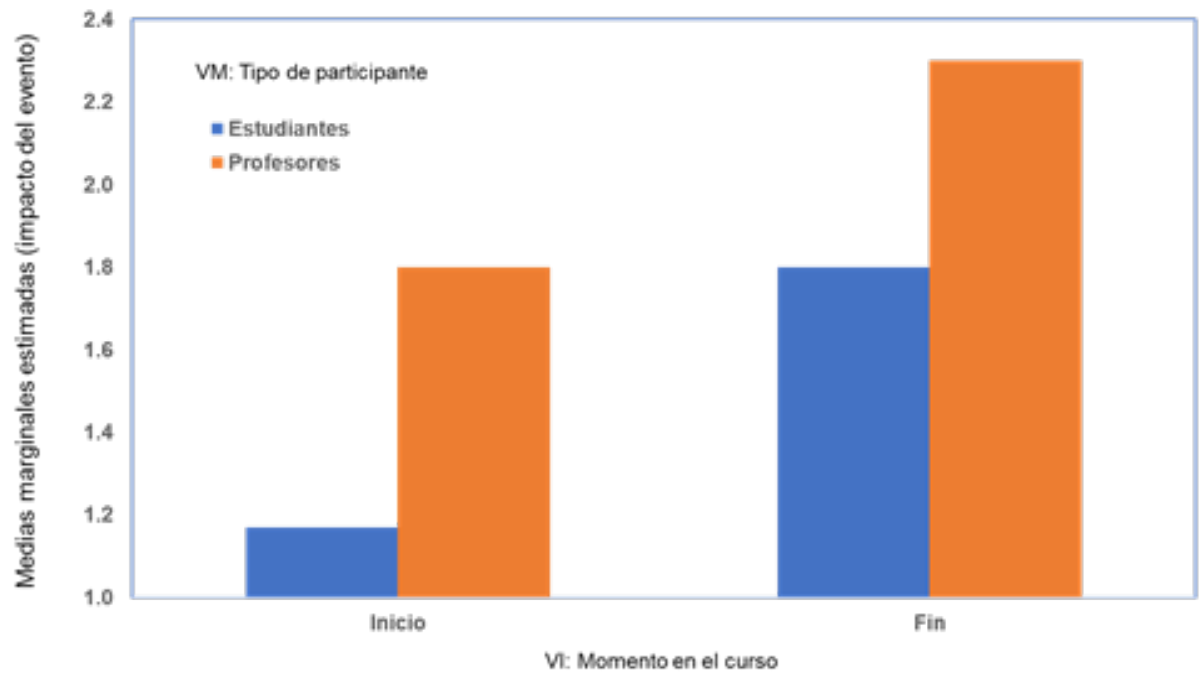

\section{Figura 5}

Análisis de moderación: perfil del participante sobre el momento del curso y la variable agotamiento $(n=834)$

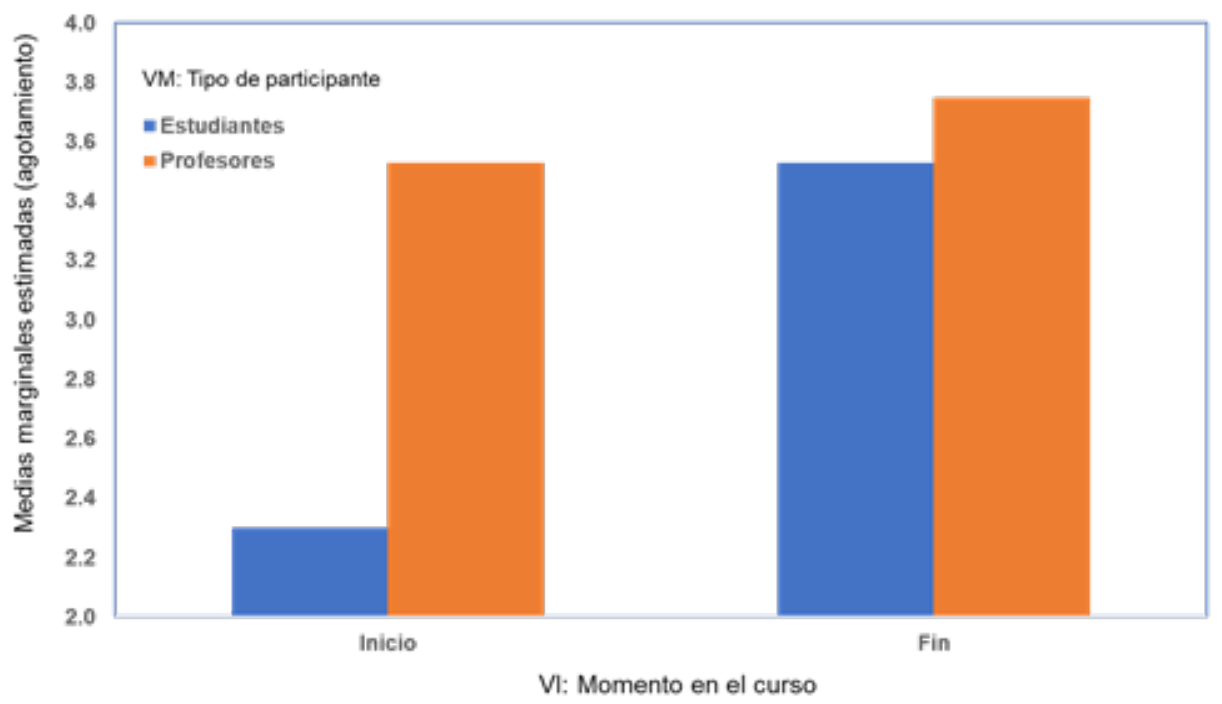




\section{Discusión}

Los resultados del estudio muestran qué antes de declarar la pandemia, al inicio del curso, en febrero de 2020, los indicadores de las variables que se asocian con la salud estaban en niveles primordialmente normales y leves. Sin embargo, una vez declarada la pandemia Covid-19 que anuncia la propagación del virus SarsCov-2, y con el primer caso detectado en a fines de febrero de 2020 en el país, el Gobierno de México, puso en marcha las medidas de distanciamiento social para evitar contagios y con ellas sus implicaciones como la falta de movilidad, confinamiento, incertidumbre sobre la salud y futuro económico. Como consecuencia del aumento de contagios y la instauración de medidas preventivas de distanciamiento, hay repercusiones en la salud mental de los participantes en el estudio -estudiantes y profesores-, tal y como se puede observar en los datos de las tablas 1, 2, 3 y 4 . Por lo que no se rechaza la $\mathrm{H} 2$ : hay manifestaciones de afectaciones a la salud mental de los alumnos y profesores como consecuencia de la pandemia Covid-19 en indicadores como la depresión, angustia, insomnio, impacto del evento Covid-19 y agotamiento. Esto en coincidencia con los estudios realizados en China en médicos y enfermeras (Wang et al., 2020; Li et al., 2020b), en pacientes con enfermedades mentales previas (Page et al., 2011) y en el meta estudio sobre brotes de epidemias virales (Ricci-Cabello et al., 2020).

La evidencia del deterioro en la salud mental de estudiantes y profesores encontrada en este estudio coincide con lo reportado por García (2020), sobre altos niveles de estrés tanto en estudiantes como en profesores, derivado de la puesta en marcha de un programa improvisado ante la emergencia, para el cual no estaban preparados, ni emocionalmente, ni con la infraestructura tecnológica para afrontar esta situación. García (2020) indica que durante el desarrollo de los cursos bajo la modalidad de enseñanza remota de emergencia algunos de los alumnos y profesores han enfrentado situaciones complejas, como lidiar con la enfermedad o bien atender familiares enfermos. Los profesores adicionalmente tuvieron que hacerse cargo en algunos casos de sus padres -personas de la tercera edad- o hijos, al mismo tiempo que adecuaban sus materiales didácticos e impartían clases, aspectos que podrían influir en el deterioro de su salud mental.

Por otro lado, se aprecia que hay una correlación positiva entre las diferentes variables de estudio de salud mental: depresión, ansiedad, insomnio, impacto del evento y agotamiento, que aumenta a medida que se extienden los casos de contagios y los días de distanciamiento social, tal y como puede apreciarse en las tablas 1 y 2 , por lo que la $\mathrm{H} 1$ no se rechaza. Esto en coincidencia con los estudios realizados en el sector salud por Wang et al., 2020 y Li et al., 2020a.

Es especialmente destacable el aumento de la correlación del impacto del evento que al inicio del curso -no pandemia-, mostraba niveles de correlación bajos con las variables de salud mental, debido a que quizá se veía lejana la posibilidad de ser afectados por la pandemia Covid-19, pero una vez que se declara ésta y se miden al final del curso las variables de salud mental, momento en que los participantes, ya han vivido la experiencia del distanciamiento social, el aumento de contagios, los efectos económicos y sociales de la pandemia, entonces la correlación de la variable impacto del evento, aumenta a niveles de correlación similares a los de las otras variables de salud mental.

En relación con el impacto de la pandemia sobre profesores y estudiantes, los datos de las tablas 3 y 4 , pusieron de manifiesto que durante la pandemia hay un mayor impacto sobre los profesores y que los estudiantes, son menos sensibles a ella. Este es un hallazgo que presenta la posibilidad de investigar la razón del 
por qué se da esta afectación en mayor medida en los profesores. Parece por la información que se obtuvo del estudio que los estudiantes son más resistentes en su salud mental al enfrentar la pandemia Covid-19. El análisis de varianza comprueba que si hay una diferencia significativa entre los efectos sufridos sobre la salud ocasionados por la pandemia entre estudiantes y profesores. Esto permite no rechazar la H3: hay una afectación diferenciada en la salud mental de alumnos y profesores como consecuencia de la pandemia Covid-19.

La razón de una afectación diferenciada podría estar sustentada en lo que García (2020) reportó en su estudio, en el que indica que cerca del $90 \%$ de los profesores a nivel de educación superior manifestaron un aumento en la carga de trabajo por adecuar sus clases ante la situación de enseñanza remota de emergencia, hacerse cargo de la familia en circunstancias de confinamiento y en algunos casos lidiar con la enfermedad, además de que en algunas situaciones vieron disminuidos sus ingresos durante el periodo de confinamiento. Por otra parte, los estudiantes reportaron que más del 80\% fueron apoyados y arropados por sus familias ante esta misma situación.

Es destacable a su vez indicar que los estudiantes tuvieron en una de las variables estudiadas un incremento importante y está fue la del agotamiento, en esta variable, los estudiantes aumentaron de manera muy importante, por lo que puede indicarse que al final del curso los estudiantes se sentían más que nada agotados por las implicaciones de estar confinados y atender sus cursos. Igualmente coincide con lo que García (2020) indica sobre los estudiantes que reportaron verse abrumados en sus cursos por el tiempo dedicado a estar frente a una pantalla, las fallas en la comunicación y la falta de contacto físico con profesores y compañeros.

Este estudio presenta resultados interesantes, pero tiene varias limitaciones y oportunidades de mejora. En primer lugar, es un estudio limitado a la comunidad académica de universidades privadas, por lo que hay un sesgo por el perfil de estudiantes y de profesores que atienden estas instituciones, por lo que valdría la pena extender el estudio a universidades públicas por una parte y a otros sectores económicos y sociales para contar con una mayor representatividad de la sociedad. Asimismo, existe la posibilidad de investigar sobre las causas que provocan la afectación a la salud mental como pueden ser el desempleo, la escasez de recursos económicos, las enfermedades de seres queridos o simplemente la incertidumbre, por lo que se abren varías posibilidades para estudiar este fenómeno.

\section{Conclusión}

En conclusión, el grupo de estudiantes y profesores de las universidades privadas sometidas a estudio durante la pandemia de Covid-19, han presentado afectaciones en su estado de salud mental como muestran las evidencias de esta investigación, ya que, las variables de estudio mostraron un cambio en los niveles moderado y severo del inicio, al final del curso, como se observa en los datos siguientes: depresión de 8.4\% a 61.5\%, ansiedad de $10.1 \%$ a $28.4 \%$, insomnio de $9.5 \%$ a $21.2 \%$, impacto del evento de $3.8 \%$ a $28.9 \%$ y agotamiento de $12.6 \%$ a $38.2 \%$.

Además, el grupo más resistente a problemas de salud mental ocasionados por la pandemia son los estudiantes y el más afectado son los profesores como se puede apreciar en los datos de las diferentes variables de estudio al final del curso en los niveles moderado y severo: depresión en estudiantes $26.5 \%$, profesores $35.7 \%$; ansiedad en estudiantes $22.4 \%$ y profesores $49.7 \%$; insomnio en estudiantes $15.8 \%$ y profesores 48.6\%; impacto del evento en estudiantes $23.0 \%$ y profesores $53.5 \%$; agotamiento en estudiantes $47.4 \%$ y 
$51.5 \%$ en profesores.

Adicionalmente los estudiantes al finalizar el curso se sentían más que nada agotados, ya que hubo un aumento importante en el nivel de moderado a severo desde el inicio del curso que se encontraba en $11.5 \%$ y pasó a $47.4 \%$.

Hasta el momento en que se realizó el estudio y con base en las conclusiones planteadas, los estudiantes muestran una mejor condición en las variables de salud mental estudiadas.

Este estudio permitió entender el impacto de la pandemia de Covid 19 en la salud mental de los estudiantes y profesores en universidades privadas. Es importante continuar con estudios en este aspecto y ampliarlo a las universidades públicas y a una escala nacional, esto podrá aportar mayores datos y entendimiento de la afectación de la pandemia del Covid 19 a una población más extensa.

Los hallazgos de este estudio pueden ayudar generar programas de acompañamiento y apoyo durante la pandemia tanto a estudiantes como a profesores, con especial énfasis a estos últimos, ya que son los más afectados, por lo que este tipo de programas pueden ser una muy buena opción de contención ante este fenómeno. El sentirse acompañado a distancia, puede ser una actividad que permita reducir la afectación en la salud mental de los profesores, el contar con apoyo pedagógico, en tecnologías de la información, psicológico y de salud, podría ayudar a reducir el nivel de afectación derivado de la pandemia. En algunas universidades como el Tecnológico de Monterrey y la Universidad Anáhuac se han desarrollado programas de acompañamiento psicológico, médico y de apoyo para compra de herramientas tecnológicas para afrontar de mejor forma la enseñanza remota de emergencia cuyos programas están disponibles en las páginas web de dichas instituciones.

En el caso del agotamiento de los estudiantes, una posibilidad, es que se desarrollen clases con mayor dinamismo en la educación a distancia, que fomenten la interacción entre los participantes mediante diálogos, dinámicas, juegos, talleres, en la que los estudiantes, interactúen, empleando la tecnología de la información para acercarse, estando a distancia y a su vez el apoyo de las universidades para que los profesores desarrollen una planeación didáctica enfocada a educación a distancia y que tanto alumnos como profesores sean capacitados para la educación a distancia.

\section{Referencias}

Baader, T., Molina, J.L., Venezian, S., Rojas, C., Farías, R., Fierro-Freixenet, C., Backenstrass, M., y Mundit, C. (2012). Validación de la encuesta PHQ-9 en el diagnóstico de depresión en pacientes usuarios de atención primaria en Chile. Revista Chilena de Neuro Psiquiatría, 50(1), 10-22.

Bastien, C, Valliéres, A., y Morin, C. (2001). Validación del índice de gravedad del insomnio como medida de resultado para investigación del insomnio. Sleep Medicine. 2(4), 297-307.

CDC (2020). Enfermedad del coronavirus 2019 (Covid-19). https://espanol.cdc.gov/coronavirus/2019-ncov/ daily-life-coping/managing-stress-anxiety.html.

Colín, C. G. (2019). El rol mediador del capital psicológico entre la adicción al trabajo y el burnout en trabajadores mexicanos. The Anáhuac Journal, 19(2), 71-96. 
https://doi.org/10.36105/theanahuacjour.2019v19n2.

Cullen, W., Gulati, G., \& Kelly, B.D. (2020). Mental health in the Covid-19 pandemic. QJM an International Journal of Medicine. 113(5), 311-312. https://doi.org/10.1093/qjmed/hcaa110

Da Silva, A.G., Miranda, D.M., Diaz, A.P, Teles, A.L.S, Malloy-Diniz, L.F., \& Palha A.P. (2020). Mental health: why it still matters in the midst of a pandemic. Brazilian Journal of Psychiatry. 42(3). 229-231. https://doi.org/1590/1516-4446-2020-0009

Duan, L., \& Zhu, G. (2020). Psychological interventions for people affectation by the Covid-19 epidemic. The Lancet, 7(4), 300-302. https://doi.org/10.1016/52215-0366(20)30073-0

ENCOVID19 (2020). Encuesta de seguimiento de los efectos del Covid en el bienestar de los hogares mexicanos. Universidad Iberoamericana. https://ibero.mx/sites/default/files/comunicadoencovid-19completo.pdf

García, L. (2021). Covid-19 y educación a distancia digital: preconfinamiento, confinamiento y posconfinamiento. RIED. Revista Iberoamericana de Educación a Distancia, 24(1), 9-32. https://doi.org/10.5944/ ried.24.1.28080

Ho, C. S., Cheen, C. Y., \& Ho, A.C. (2020). Mental health strategies to combat the psychological impact of Covid-19 beyond paranoia and panic. Annals of the Academy of Medicine of Singapore, 49(3), 155160.

Hodges, C., Moore, S., Lockee, B., Trust, T., \& Bond, A. (2020). The difference between remote teaching and online learning. Educase Review. https://er.educase.edu/articles(2020/3/the-difference-betweenemergency-remote-teaching-and-onlinelearning

Hu, L. S., \& Bentler, P. M. (1999). Cuttoff criteria for fit indexes in covariance structure analysis: Conventional criteria versus new alternatives. Structural Equation Modeling; A Multidisciplinary Journal, 6, 1-55. https://doi.org/101080/107055199095400118

IESALC-UNESCO (2020). El coronavirus-19 y la educación superior: impacto y recomendaciones. https:// cutt.ly/xdHJuhK

Jakovljevic, M., Bjedov, S. Jaksic, N., \& Jakovljevic, I. (2020). Covid-19 pandemia and public and global mental health form the perspective of global health security. Psychiatria Danubina, 32(1), 6-4. https:// doi.org/10.24869/psyd.2020.6

Levy, S. (2020). Covid-19 Serie de documento de política pública: sugerencias para la emergencia. PNUD $L A C$ C19 PDS, 2,

Li, J., Ma. S., Wang., Y., Cai, Z., Hu, J., Wei, N., Du, H., Chen, T., Li, R., Tan, H., Kang, L., Yan., L., Huang, M., Wang, H., Wang, G., Liu, Z., \& Shauhua, H. (2020a). Factors associated with mental health outcomes among health care workers exposed to Coronavirus disease 2019. JAM Network Open, 3(3): e203976. https://doi.org/10.1001/jamanetworkopen.2020.3976

Li, W., Yang, Y, Liu, Z., Zhao, Y., Zhang, Q., Zhang, L., Cheung, T., \& Xiang, Y. (2020b). Progression on 
mental Health Services during the COVID-19 Outbreak in China. International Journal of Biological Sciences, 16(10), 1732-1738. https://doi.org/10.7150/ijbs.45120

Maslach, C., \& Jackson, S. E. (1981). The measurement of experienced burnout. Journal of Organizational Behavior, 2(2), 99-113.

Means, B., Bakia, M., y Murphy, R. (2014). Aprender en línea: lo que la investigación nos dice sobre sí, cuándo y cómo. Routledge.

OMS (2020). Covid-19: Cronología de la actuación de la OMS. https://www.who.int/es/news-room/detai1/27-04-2020-who-timeline---covid.19

OMS (2005). Alerta epidémica y respuesta. Plan mundial de las OMS de preparación para una pandemia de influenza: función y recomendaciones de la OMS para las medidas nacionales antes y durante la pandemia. https://www.paho.org/spanish/ad/dpc/cd/vir-flu-plan-mundial-oms.htm

Page, L.A., Seetharaman, S., Suhail, I., Wesseley, S., Pereira, J., \& Rubin, J.G. (2011). Using electronic patient records to assess the impact of swine flue (Influenza H1N1) on mental health patients. Journal of Mental Health, 20(1), 60-69.

Pérez, C. (2004). Técnicas de análisis multivariado de datos. Pearson-Prentice Hall.

Podsakoff, P., Mackenzie, S., Lee, J., \& Podsakoff, N. (2003). Common method biases in behavioral research: a critical review of the literature and recommended remedies. Journal of Applied Psychology, 88(5), 879-903. https://doi.org/10.1037/0021-9010.88.5.879

Ricci-Cabello, I., Meneses-Echavez. J.F., Serrano-Ripoll, M.J., Fraile-Navarro, D., Fiol de Roque, M.A., Pastor, G., Castro, A., Ruiz-Perez, I., Zamanillo, R., y Goncalves-Bradley, D. (2020). Impact of viral epidemic outbreaks on mental health of healthcare workers: A rapid systemic review. medRxiv, preprint. https://doi.org/10.1101/2020.04.02.20048892

Secretaría de Salud (2020). Todo sobre el Covid-19. Recuperado de https://coronvirus.gob.mx

Spitzer, R.L., Kroenke, K., Williams, J.B.W., Löwe, B. (2006). A Brief Measure for Assessing Generalizad Anxiety Disorder: The GAD-7. Archives of Internal Medicine, 166(10), 1092-1097

Taylor, S. (2019). The Psychology of Pandemics: Preparing for the next Global Outbreak of Infectious Disease. Newcastle upon Tyne, Cambridge Scholars Publishing.

UNESCO (2020). Startling digital divides in distance learning emerge. Recuperado de https://cutt.ly/adH2S$\underline{\mathrm{RS}}$

Wang, C., Pan, R., Wang, X., Tan, Y., Xu, L. y Ho, C.S. (2020). Immediate psychological responses and associated factors during the initial stage of the 2019 coronavirus disease (COVID-19) epidemic among the general population in China. International Journal of Environment Research on Public Health, 17, $17-29$

Weiss, D. \& Marmar, C. (1997). The impact of event scale-revised. En J. Wilson \& M. Keane (Eds)., Assessing Psychological Trauma and PTSD. Guilford Press. 